\title{
Preoperative Risk Stratification of Endometrial Carcinoma : L1CAM as a Biomarker
}

\section{Pasanen, Annukka}

2017-09

Pasanen, A , Loukovaara , M , Tuomi , T \& Butzow , R 2017 , ' Preoperative Risk Stratification of Endometrial Carcinoma : L1CAM as a Biomarker ' , International Journal of Gynecological Cancer , vol. 27 , no. 7 , pp. 1318-1324 . https://doi.org/10.1097/IGC.0000000000001043

http://hdl.handle.net/10138/311349

https://doi.org/10.1097/IGC.0000000000001043

cc_by_nc

acceptedVersion

Downloaded from Helda, University of Helsinki institutional repository.

This is an electronic reprint of the original article.

This reprint may differ from the original in pagination and typographic detail.

Please cite the original version. 


\section{Preoperative risk stratification of endometrial carcinoma: LI cell \\ 2 adhesion molecule as a biomarker}

3

4

5

6

Objective: Pre- or intraoperative risk assessment models are used to stratify patients with endometrial carcinoma to lymphadenectomy. Our aim was to determine whether preoperative analysis of $\mathrm{L} 1$ cell adhesion molecule (L1CAM) can improve risk assessment.

Methods: Immunohistochemical L1CAM staining was performed on endometrial biopsies of 241 patients and paired hysterectomy samples of 75 patients. Risk assessment models based on preoperative histological type and grade, myometrial invasion and/or tumor diameter and alternative models incorporating preoperative L1CAM were compared with regard to their capability of predicting lymph nodal or distant metastasis. Soluble L1 levels were measured by ELISA in serum samples of 40 patients with endometrial carcinoma.

Results: The concordance rate between L1CAM staining results of preoperative and hysterectomy samples was moderate (kappa 0.586, $P$ < 0.0001). Preoperative L1CAM expression was associated with non-endometrioid histology, lymph node involvement, advanced stage and positive peritoneal cytology. Receiver operating characteristic (ROC) analyses showed that L1CAM did not significantly improve risk stratification algorithms based on traditional risk factors. Intraoperative tumor diameter was an effective surrogate for myometrial invasion. There was no statistical difference between $\mathrm{L} 1$ serum levels of patients with a L1CAM-positive or L1CAM-negative endometrial carcinoma $(P=0.786)$.

Conclusions: L1CAM expression in endometrial biopsy correlates with high risk features of endometrial carcinoma but does not significantly improve risk stratification algorithms based on traditional factors. Soluble L1 detected in the serum of patients with endometrial carcinoma does not correlate with tumoral L1CAM expression.

Keywords endometrial cancer, risk stratification, lymphadenectomy, L1CAM, soluble L1CAM 
32 The standard primary treatment of endometrial carcinoma consists of surgery with total

33 hysterectomy and bilateral salpingo-oophorectomy, complemented with pelvic and para-aortic

34 lymphadenectomy in selected cases [1]. There is no compelling evidence that lymphadenectomy as such is therapeutically beneficial and its main motivation is to more accurately stage and stratify patients to postoperative adjuvant therapy. However, extensive surgery may cause morbidity and the rate of unnecessary treatment should be minimized [2]. At most institutions the decision on lymphadenectomy is made pre- or intraoperatively based on features of the primary tumor, including histologic type, grade of differentiation, and depth of myometrial invasion, as evaluated by preoperative histology, frozen section analysis and imaging.

The most validated algorithm (so called Mayo criteria, [3]) defines low risk endometrial carcinoma as endometrioid G1-2 carcinoma with tumor diameter $\leq 2 \mathrm{~cm}$ and myometrial invasion $\leq 50 \%$.

When this algorithm is applied, approximately $30 \%$ of the patients fall into the category of low risk for lymphatic dissemination and may avoid lymphadenectomy [4,5]. The remaining $70 \%$ of the patients should undergo lymphadenectomy, yet only 19-22\% of them present with lymph node metastases $[4,5]$. More recently a risk stratification model was presented, according to which over $40 \%$ of the patients could be spared lymphadenectomy with a false positive rate of $57.2 \%$ and false negative rate of $0 \%$ [6]. This model is based on tumor grade (G1-2 vs G3), diameter (50-mm cutoff) and depth of myometrial invasion (MI, three-tiered). A major difficulty related to these algorithms is the often inaccurate assessment of myometrial invasion by preoperative imaging or gross visualization [7-9]. Frozen section diagnosis is not readily available in many institutions [10] and various investigators question its accuracy [11-13]. Intraoperatively assessed tumor diameter has been proposed as a surrogate for myometrial invasion $[6,14,15]$ 
54 Current risk assessment algorithms are burdened with a high frequency of presumably unnecessary

55 lymphadenectomies. To overcome this problem, attention has been paid to the potential value of molecular markers (such as ER/PR status in predicting lymph node involvement [16]).

57 Nevertheless, molecular markers do not have an established role in this setting nor have they been 58 integrated in randomized clinical trials of surgical therapies.

59 A promising prognostic marker, L1 cell adhesion molecule (L1CAM, CD171) predicts disease

60

61

62

63

64 progression and poor prognosis in many types of cancer including endometrial carcinoma [17-23]. The association between L1CAM expression and lymph node involvement of endometrial carcinoma suggests that L1CAM could be a useful biomarker for stratifying patients to lymphadenectomy [21-23]. Also, a soluble form of L1CAM (sL1) exists and has been detected in the serum and ascites of patients carrying a tumor expressing this antigen [17,24-26].

Based on the association of L1CAM expression with lymphatic dissemination in endometrial carcinoma, we wanted to evaluate the power of L1CAM in algorithms aimed at stratifying patients to lymph node dissection. To further clarify the potentiality of L1CAM as a biomarker, we compared serum L1CAM concentrations in patients with negative and positive L1CAM expression in tumor sections.

\section{Material and methods}

Patients who underwent primary surgical treatment for endometrial carcinoma at the Department of 4 Obstetrics and Gynecology, Helsinki University Hospital, between January 1, 2007 and December 31, 2009 were identified. Patients with a preoperative endometrial sample available for L1CAM analysis were included in the study $(n=241)$. Approvals of the Institutional Review Board and the 7 National Authority for Medicolegal Affairs of Finland were obtained. During 2007-09, according to 
the treatment guidelines of our hospital, bilateral pelvic lymphadenectomy was performed in patients with grade 1-2 endometrioid carcinoma with $<50 \%$ myometrial invasion, the depth of invasion being assessed by vaginal ultrasound and gross visual inspection. In other patients, both pelvic and paraaortic lymphadenectomies were performed. There was some variation in practice patterns because the decision to perform lymphadenectomy and the extent of the procedure depended on patient age and surgical risks. Total rate of lymphadenectomy was $79.7 \%$. Pertinent patient characteristics and surgical data are shown in Table 1.

Factors selected for statistical analyses were: preoperative L1CAM expression, FIGO 2009 stage [27], lymph node involvement, histologic type (endometrioid/non-endometrioid), grade of differentiation, depth of myometrial invasion, tumor diameter, cervical stromal invasion, peritoneal cytology status, patient age at surgery, and body mass index (BMI). The cut off values for the numeric variables $(\geq 50 \%$ and $>33 \%$ for myometrial invasion, $2 \mathrm{~cm}$ and $5 \mathrm{~cm}$ for tumor diameter, 65 years for age, $30 \mathrm{~kg} / \mathrm{m}^{2}$ for BMI) were based on earlier reports [6,28-30]. Tumor size was measured intraoperatively by the surgeon or after formaldehyde fixation by the pathologist. Primary tumor diameter was defined as the largest dimension of the tumor. If more than 1 lesion was present, the lesion with the largest diameter was considered. Primary tumor diameter was unknown in 14 patients. The presence of cervical stromal invasion was unknown in 2 patients. Peritoneal cytology was considered positive if adenocarcinoma cells were detected in the peritoneal washing, regardless of the number of cancer cells. One case that was positive due to a concomitant borderline serous ovarian tumor was considered negative for endometrial cancer. Peritoneal cytology status was unknown in 4 patients.

Preoperative L1CAM staining was assessed in tissue samples obtained by uterine aspiration biopsy or curettage. Uterine biopsy was the primary (>90\%) sampling method. Uterine curettage was performed when biopsy was insufficient for diagnosis or failed due to cervical stenosis. For immunohistochemical stainings, slides were stained with Ventana Benchmark XT automated slide 
preparation system (Ventana Medical Systems, Inc., USA) or with Autostainer LV1 (Lab Vision

104 Corporation, USA). Briefly, slides were deparaffinized and heat-induced epitope retrieval was performed following standard protocol. Tissue sections were incubated with primary monoclonal antibodies against L1CAM (CD171; clone 14.10, catalog number SIG-3911-1000, Covance Inc., NJ, USA). The antibody binding site was visualized using a DAB Detection Kit. Sections were counterstained with Mayer's hematoxylin, dehydrated, cleared in xylene, and mounted. L1CAM positivity was defined as $>10 \%$ of the carcinoma cells staining in one representative slide evaluated by a pathologist (Supplementary figure 1). Neural cells of an appendix slide served as an external positive control and myometrial nerves as an internal positive control (for whole sections). For concordance studies we stained the corresponding hysterectomy sections of all the patients with a positive $(\mathrm{n}=50)$ and of 25 patients with a negative preoperative sample.

Starting from November 2014, we have obtained a preoperative blood sample from voluntary patients with endometrial carcinoma treated at the Department of Obstetrics and Gynecology, Helsinki University hospital. Blood fractionation was carried out by centrifugation for $10 \mathrm{~min}$ at $2000 \times \mathrm{g}$ and the samples were stored at $-70^{\circ} \mathrm{C}$. The serum samples of all the patients with an immunohistochemically verified L1CAM-positive $(\mathrm{n}=17)$ and 23 patients with an L1CAMnegative endometrial carcinoma were retrieved. To determine the serum level of L1CAM we used a commercial enzyme linked immunosorbent assay (ELISA) kit (LifeSpan Biosciences Inc., WA, USA, Catalog No. LS-F24209). Standards, controls and samples were processed for sandwich ELISA according to the manufacturer's instructions and duplicate wells were ran for each sample. Final serum dilution (1:2000) was chosen after running test reactions on serial dilutions. The absorbance at $450 \mathrm{~nm}$ was measured by an automatic ELISA reader (Multiskan EX, Thermo Fisher Scientific, USA). Results were expressed in $\mathrm{ng} / \mathrm{ml}$ according to the established standard curve. The limit of detection was $93.75-6000 \mathrm{pg} / \mathrm{ml}$. 
analyses were used to compute odds ratios (OR) along with $95 \%$ confidence intervals $(\mathrm{CI})$ for the

129

130

131

132

133

134

135

136

137

\section{Results} associations between preoperative L1CAM staining and various risk parameters in the cohort. Multiple regression analysis was used to estimate the independent effect of selected risk parameters on either preoperative L1CAM staining or lymph node/distant metastasis (stage IIIC-IV disease). Cohen's kappa statistics were calculated to measure the agreement of preoperative L1CAM staining and tumor histology with corresponding postoperative findings. Based on kappa references outlined by Landis and Koch [31], the strength of agreement was considered moderate for kappa values between 0.41 and 0.60 and substantial for kappa values between 0.61 and 0.80 .

Multivariable models were created to test the capability of preoperative L1CAM to predict lymph node and distant metastasis in conjunction with other risk parameters. The estimated weight of each parameter included in a risk model, was determined by rounding statistically significant odds ratios in the multivariable models to the nearest integer. These risk points of each factor were summed to generate a risk score potentially predicting the probability of advanced disease. The risk scores were used to test the discriminating abilities of the risk models with the 2tailed receiver operating characteristic (ROC) curve area comparison test. Alternative models were created by eliminating selected variables from the models. Statistical significance was set at $\mathrm{P}<0.05$. Data were analyzed using IBM SPSS version 22 software (IBM Corp., Armonk, NY, USA).

Of the 241 preoperative endometrial samples, 64 (26.6\%) were L1CAM positive. L1CAM expression was observed in $22.3 \%$ (43/193) of grade 1-2 endometrioid carcinomas, $27.6 \%(8 / 29)$ of grade 3 endometrioid carcinomas, and $68.4 \%(13 / 19)$ of non-endometrioid carcinomas $(\mathrm{P}<0.0001)$. According to kappa statistics in 75 sample pairs, preoperative L1CAM staining showed moderate 
152

153

154

155

156

157

158

159

160

161

162

163

164

165

166

167

168

169

170

171

172

173

174

175

176

agreement with findings in the whole section (kappa 0.586, $\mathrm{P}<0.0001$ ). By comparison, in the whole study population of 241 patients, kappa value was $0.551(\mathrm{P}<0.0001)$ for the agreement of preoperative histology with final histology in detecting high risk cases (grade 3 or non-endometrioid carcinoma). We did not observe any special L1CAM staining pattern, such as preferential positivity at the myoinvasive front, in the whole sections of hysterectomy specimens.

Preoperative L1CAM positivity was associated with disease spread beyond the uterine corpus, lymph node involvement, non-endometrioid histology, positive peritoneal cytology, and high age (Table 2). Logistic regression analysis indicated that non-endometrioid histology was independently associated with L1CAM positivity, whereas the effect of disease spread beyond uterine corpus, positive peritoneal cytology or high age was not significant (Table 3). Preoperative high risk histology (grade 3 or non-endometrioid carcinoma), myometrial invasion ( $>33 \%$ or $\geq 50 \%$ ), tumor diameter ( $\geq 2 \mathrm{~cm}$ or $\geq 5 \mathrm{~cm})$ and preoperative L1CAM positivity were included in logistic regression models, with lymph node and distant metastasis as the dependent variable. Patients with available data for all the variables were included in each model $(n \geq 225)$. Tumor size $\geq 2 \mathrm{~cm}$ was the only variable that failed to display a significant independent effect on the dependent variable (Table 4). Addition of L1CAM in the models did not significantly improve the AUCs of the risk stratification algorithms $(\mathrm{P}>0.28$, Table 5). Elimination of myometrial invasion from Cox Bauer's model (TD $\geq 5$ $\mathrm{cm}, \mathrm{MI}>33 \%)$, did not significantly diminish the AUC of the score $(\mathrm{P}=0.429)$.

There was no statistically significant difference between the concentrations of soluble L1 (s-L1) in the serum samples of patients with L1CAM positive or negative tumors $(\mathrm{P}=0.786)$. The mean $( \pm$ SD) soluble L1 concentration was $3235.49 \pm 808.60 \mathrm{ng} / \mathrm{ml}$ for L1CAM positive cases and $3163.27 \pm$ $765.90 \mathrm{ng} / \mathrm{ml}$ for L1CAM negative cases. Median (25th and 75th percentiles) soluble L1 values were 3033.90 (2680.60 and 3637.60 respectively) $\mathrm{ng} / \mathrm{ml}$ in the patients with L1CAM positive tumor and 2992.20 (2649.75 and 3467.10 respectively) $\mathrm{ng} / \mathrm{ml}$ in the L1CAM negative controls. 


\section{Discussion}

178 Modern management of endometrial cancer is based on personalized surgical and adjuvant treatment.

179 Reliable pre- or intraoperative risk stratification plays a key role in tailoring optimal surgical 180 treatment. Currently used risk assessment methods suffer from inaccuracy and definite indications

181 for lymphadenectomy are yet to be established.

182 L1CAM is a promising prognostic marker that independently predicts poor outcome and lymph nodal involvement in endometrial carcinoma [21-23]. Since L1CAM expression pattern is heterogeneous in endometrial carcinoma (10-100\% of the carcinoma cells staining in a positive immunohistochemical assay) and endometrial aspiration biopsy represents only a small portion of the tumor, the true value of L1CAM as a preoperative marker has to be studied on preoperative diagnostic samples. Despite the heterogeneous staining pattern of L1CAM, we observed a moderate concordance rate (kappa 0.586, $\mathrm{P}<0.0001$ ) between L1CAM staining in preoperative and hysterectomy samples. It is noteworthy, that the concordance between pre- and postoperative L1CAM staining was superior compared to the concordance of pre- and postoperative histology (low vs high grade). L1CAM expression was associated with disease spread beyond uterine corpus $(\mathrm{OR} 2.5, \mathrm{P}=$

192 0.003), but its significant effect was lost once other factors were taken into account. Further, L1CAM did not significantly improve the performance of risk assessment algorithms based on traditional risk factors. These results imply, that L1CAM is not a useful tool for preoperative treatment planning of endometrial carcinoma.

Considering the common difficulties in assessing the depth of myometrial invasion preoperatively, we wanted to test a model without MI as a parameter. Intraoperative tumor diameter is a more feasible measure since it can be reliably evaluated by gross inspection (by the surgeon) even when frozen section analysis is not available. In our study cohort the risk assessment model presented by Cox Bauer et al. performed equally well independently of the presence of MI as a parameter (AUC > 0.8; $\mathrm{P}=0.429$ ), suggesting that intraoperative tumor diameter could be used as an alternative to MI to 
identify high risk disease, as indicated by previous studies $[14,15]$. The ideal cut off value for tumor diameter that determines high risk disease needs to be established by further studies.

No serum markers have any established role in the treatment of endometrial carcinoma. Few studies have addressed the potential clinical usefulness of serum L1CAM. Fogel et al. detected sL1 in the blood of patients with an advanced L1CAM-positive ovarian or uterine carcinoma, but not in healthy subjects or patients with other types of tumors, suggesting that sL1 could be used in diagnostics or follow up of ovarian and uterine carcinoma [17]. Using a commercial ELISA-kit optimized for serum samples, we were not able to confirm the results of the earlier report. Based on our results, soluble L1CAM is not a useful marker of L1CAM positivity of endometrial carcinoma.

A strength of our study was its unselected cohort of patients with endometrial carcinoma treated at a single tertiary care center with well-defined diagnostic and operative standards and systematic follow-up procedures. The relatively high lymphadenectomy rate $(192 / 241,79.7 \%)$ in the study cohort improved the diagnostics of occult nodal disease permitting more accurate staging. In our institution frozen section is not used to determine the depth of MI and data on MI had to be extrapolated from final pathological reports.

In summary, we found a moderate concordance for L1CAM status between endometrial biopsies and corresponding hysterectomy specimens. Preoperative L1CAM expression was associated with lymph nodal and distant metastasis, but L1CAM did not significantly improve risk stratification algorithms based on preoperative histology, tumor diameter and/or myometrial invasion. Interestingly, the performance of risk stratification models did not depend on the presence of myometrial invasion as a variable, suggesting that the more feasible tumor diameter could be used as a surrogate variable. Based on our results, preoperative L1CAM cannot be recommended as a tool for stratifying patients with endometrial carcinoma to lymphadenectomy. 
Figure legend

Supplementary figure 1 .

232

233

234

235

236

237

238

239

240

241

242

243

244

245

246

247

248

249

250

251

252

253

254

255

256

257

258

259

260

261

262

263

264

265

Figure 1. L1CAM immunohistochemical staining patterns in biopsies containing endometrial carcinoma. a) diffuse; $b, c)$ heterogeneous ( $b$ and $c$ from the same biopsy)

[1] Burke WM, Orr J, Leitao M, Salom E, Gehrig P, Olawaiye AB, et al. Endometrial cancer: A review and current management strategies: Part I. Gynecol.Oncol. 2014 8;134:385-392.

[2] May K, Bryant A, Dickinson HO, Kehoe S, Morrison J. Lymphadenectomy for the management of endometrial cancer. Cochrane Database Syst.Rev. 2010 Jan 20;(1):CD007585. doi:CD007585.

[3] Mariani A, Webb MJ, Keeney GL, Haddock MG, Calori G, Podratz KC. Low-risk corpus cancer: Is lymphadenectomy or radiotherapy necessary? Obstet.Gynecol. 2000 6;182:15061519.

[4] Mariani A, Dowdy SC, Cliby WA, Gostout BS, Jones MB, Wilson TO, et al. Prospective assessment of lymphatic dissemination in endometrial cancer: a paradigm shift in surgical staging. Gynecol.Oncol. 2008 Apr;109:11-18.

[5] Kumar S, Podratz KC, Bakkum-Gamez JN, Dowdy SC, Weaver AL, McGree ME, et al. Prospective assessment of the prevalence of pelvic, paraaortic and high paraaortic lymph node metastasis in endometrial cancer. Gynecol.Oncol. 2014 1;132:38-43.

[6] Cox Bauer CM, Greer DM, Kram JJ, Kamelle SA. Tumor diameter as a predictor of lymphatic dissemination in endometrioid endometrial cancer Gynecol.Oncol. 2016 May;141:199-205.

[7] Mavromatis ID, Antonopoulos CN, Matsoukis IL, Frangos CC, Skalkidou A, Creatsas G, et al. Validity of intraoperative gross examination of myometrial invasion in patients with endometrial cancer: a meta-analysis Acta Obstet.Gynecol.Scand. 2012 Jul;91:779-793. [8] Antonsen SL, Jensen LN, Loft A, Berthelsen AK, Costa J, Tabor A, et al. MRI, PET/CT and ultrasound in the preoperative staging of endometrial cancer - a multicenter prospective comparative study. Gynecol.Oncol. 2013 Feb;128:300-308.

[9] Alcazar JL, Dominguez-Piriz J, Juez L, Caparros M, Jurado M. Intraoperative Gross Examination and Intraoperative Frozen Section in Patients With Endometrial Cancer for Detecting Deep Myometrial Invasion: A Systematic Review and Meta-analysis Int.J.Gynecol.Cancer 2016 Feb;26:407-415. 
[10] Soliman PT, Frumovitz M, Spannuth W, Greer MJ, Sharma S, Schmeler KM, et al. Lymphadenectomy during endometrial cancer staging: Practice patterns among gynecologic oncologists. Gynecol.Oncol. 2010 11;119:291-294. section analyses as predictors of lymphatic spread in patients with early-stage uterine cancer J.Am.Coll.Surg. 2004 Sep;199:388-393.

[12] Case AS, Rocconi RP, Straughn Jr. JM, Conner M, Novak L, Wang W, et al. A prospective blinded evaluation of the accuracy of frozen section for the surgical management of endometrial cancer. Obstet.Gynecol. 2006;108:1375-1379.

[13] Kumar S, Bandyopadhyay S, Semaan A, Shah JP, Mahdi H, Morris R, et al. The role of frozen section in surgical staging of low risk endometrial cancer. PLoS One 2011;6:e21912. [14] AlHilli MM, Podratz KC, Dowdy SC, Bakkum-Gamez JN, Weaver AL, McGree ME, et al. Preoperative biopsy and intraoperative tumor diameter predict lymph node dissemination in endometrial cancer. Gynecol.Oncol. 2013 Feb;128:294-299.

[15] Tuomi T, Pasanen A, Luomaranta A, Leminen A, Butzow R, Loukovaara M. Riskstratification of endometrial carcinomas revisited: A combined preoperative and intraoperative scoring system for a reliable prediction of an advanced disease. Gynecol.Oncol. 2015 Apr;137:23-27.

[16] Trovik J, Wik E, Werner HM, Krakstad C, Helland H, Vandenput I, et al. Hormone receptor loss in endometrial carcinoma curettage predicts lymph node metastasis and poor outcome in prospective multicentre trial Eur.J.Cancer 2013 Nov;49:3431-3441.

[17] Fogel M, Gutwein P, Mechtersheimer S, Riedle S, Stoeck A, Smirnov A, et al. L1 expression as a predictor of progression and survival in patients with uterine and ovarian carcinomas. The Lancet 2003 9/13;362:869-875.

[18] Zeimet AG, Reimer D, Huszar M, Winterhoff B, Puistola U, Azim SA, et al. L1CAM in early-stage type I endometrial cancer: results of a large multicenter evaluation. J.Natl.Cancer Inst. 2013 Aug 7;105:1142-1150.

[19] Bosse T, Nout RA, Stelloo E, Dreef E, Nijman HW, Jürgenliemk-Schulz IM, et al. L1 cell adhesion molecule is a strong predictor for distant recurrence and overall survival in early stage endometrial cancer: Pooled PORTEC trial results. Eur.J.Cancer 2014 10;50:2602-2610. [20] Altevogt P, Doberstein K, Fogel M. L1CAM in human cancer Int.J.Cancer 2015 Jun 25. [21] Dellinger TH, Smith DD, Ouyang C, Warden CD, Williams JC, Han ES. L1CAM is an independent predictor of poor survival in endometrial cancer - An analysis of The Cancer Genome Atlas (TCGA) Gynecol.Oncol. 2016 May;141:336-340.

[22] van der Putten LJ, Visser NC, van de Vijver K, Santacana M, Bronsert P, Bulten J, et al. L1CAM expression in endometrial carcinomas: an ENITEC collaboration study Br.J.Cancer 2016 Aug 9.

[23] Pasanen A, Tuomi T, Isola J, Staff S, Butzow R, Loukovaara M. L1 Cell Adhesion Molecule as a Predictor of Disease-Specific Survival and Patterns of Relapse in Endometrial Cancer Int.J.Gynecol.Cancer 2016 Oct;26:1465-1471.

[24] Gutwein P, Stoeck A, Riedle S, Gast D, Runz S, Condon TP, et al. Cleavage of L1 in exosomes and apoptotic membrane vesicles released from ovarian carcinoma cells Clin.Cancer Res. 2005 Apr 1;11:2492-2501.

[25] Zander H, Rawnaq T, von Wedemeyer M, Tachezy M, Kunkel M, Wolters G, et al. Circulating levels of cell adhesion molecule $\mathrm{L} 1$ as a prognostic marker in gastrointestinal stromal tumor patients. BMC Cancer 2011 May 22;11:189:1-7.

[26] Aktas B, Kasimir-Bauer S, Wimberger P, Kimmig R, Heubner M. Utility of mesothelin, L1CAM and Afamin as biomarkers in primary ovarian cancer Anticancer Res. 2013 Jan;33:329-336. 
[27] Pecorelli S. Revised FIGO staging for carcinoma of the vulva, cervix, and endometrium.

316 Int.J.Gynaecol.Obstet. 2009 May;105:103-104.

[28] Milam MR, Java J, Walker JL, Metzinger DS, Parker LP, Coleman RL, et al. Nodal metastasis risk in endometrioid endometrial cancer. Obstet.Gynecol. 2012 Feb;119:286-292. [29] Benedetti Panici P, Basile S, Salerno MG, Di Donato V, Marchetti C, Perniola G, et al. Secondary analyses from a randomized clinical trial: age as the key prognostic factor in endometrial carcinoma. Am.J.Obstet.Gynecol. 2014 Apr;210:363.e1-363.e10. [30] Secord AA, Hasselblad V, Von Gruenigen VE, Gehrig PA, Modesitt SC, Bae-Jump V, et al. Body mass index and mortality in endometrial cancer: A systematic review and metaanalysis. Gynecol.Oncol. 2016 Jan;140:184-190.

[31] Landis JR, Koch GG. The measurement of observer agreement for categorical data. 

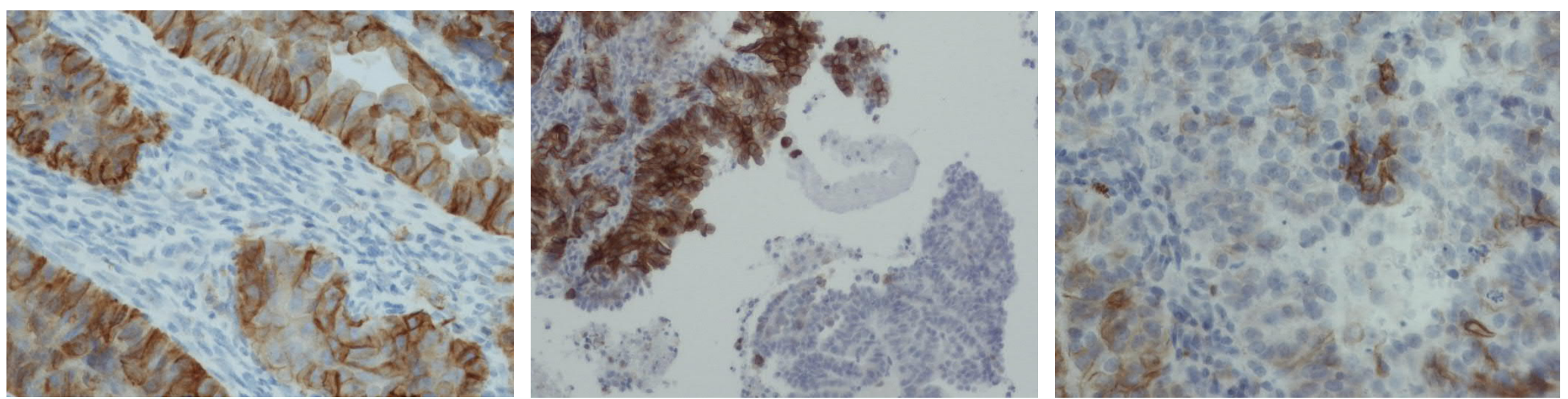

Figure 1. L1CAM immunohistochemical staining patterns in biopsies containing endometrial carcinoma. a) diffuse, $b, c$ ) heterogeneous ( $b$ and $c$ from the same biopsy) 
Table 1. Clinicopathologic data $(n=241)$.

\begin{tabular}{|c|c|}
\hline Age (years) $($ mean \pm SD) & $67.3 \pm 10.4$ \\
\hline Body mass index $\left(\mathrm{kg} / \mathrm{m}^{2}\right)($ mean $\pm \mathrm{SD})$ & $28.5 \pm 6.5$ \\
\hline Pelvic lymphadenectomy (number of cases, percent) & $162(67.2 \%)$ \\
\hline Pelvic-aortic lymphadenectomy (number of cases, percent) & $30(12.4 \%)$ \\
\hline \multicolumn{2}{|l|}{ Adjuvant therapy (number of cases, percent) } \\
\hline Vaginal brachytherapy & $133(55.2 \%)$ \\
\hline Whole pelvic radiotherapy & $28(11.6 \%)$ \\
\hline Chemotherapy & $6(2.5 \%)$ \\
\hline Chemotherapy and vaginal brachytherapy & $13(5.4 \%)$ \\
\hline Chemotherapy and whole pelvic radiotherapy & $33(13.7 \%)$ \\
\hline \multicolumn{2}{|l|}{ Histology (number of cases, percent) } \\
\hline Endometrioid carcinoma & $222(92.1 \%)$ \\
\hline Clear cell carcinoma & $7(2.9 \%)$ \\
\hline Serous carcinoma & $4(1.7 \%)$ \\
\hline Undifferentiated carcinoma & $2(0.8 \%)$ \\
\hline Carcinosarcoma & $5(2.1 \%)$ \\
\hline Neuroendocrine carcinoma & $1(0.4 \%)$ \\
\hline \multicolumn{2}{|l|}{ Grade (number of cases, percent) (For endometrioid only, $n=222$ ) } \\
\hline Grade 1 & $128(57.7 \%)$ \\
\hline Grade 2 & $65(29.3 \%)$ \\
\hline Grade 3 & $29(13.1 \%)$ \\
\hline \multicolumn{2}{|l|}{ FIGO 2009 stage (number of cases, percent) } \\
\hline IA & $130(53.9 \%)$ \\
\hline IB & $54(22.4 \%)$ \\
\hline II & $13(5.4 \%)$ \\
\hline IIIA & $12(5.0 \%)$ \\
\hline IIIB & $1(0.4 \%)$ \\
\hline IIIC1 & $18(7.5 \%)$ \\
\hline IIIC2 & $7(2.9 \%)$ \\
\hline IVA & $0(0 \%)$ \\
\hline IVB & $6(2.5 \%)$ \\
\hline
\end{tabular}


Table 2. Clinicopathologic characteristics according to L1CAM expression in preoperative endometrial samples, univariate analysis

\begin{tabular}{|l|l|l|l|l|}
\hline Variable & Negative L1CAM & Positive L1CAM & OR (95\% CI) & P \\
\hline Stage II-IV & $34 / 177(19.2 \%)$ & $24 / 64(37.5 \%)$ & $2.5(1.3-4.7)$ & 0.003 \\
\hline Positive pelvic and/or para- & $12 / 174(6.9 \%)$ & $13 / 61(21.3 \%)$ & $3.7(1.6-8.5)$ & 0.002 \\
aortic lymph nodes a & & & & \\
\hline Non-endometrioid carcinoma & $6 / 177(3.4 \%)$ & $13 / 64(20.3 \%)$ & $7.3(2.6-20)$ & $<0.0001$ \\
\hline Grade 3 (endometrioid only) & $21 / 171(12.3 \%)$ & $8 / 51(15.7 \%)$ & $1.3(0.55-3.2)$ & 0.526 \\
\hline Myometrial invasion $\geq 50 \%$ & $71 / 177(40.1 \%)$ & $27 / 64(42.2 \%)$ & $1.1(0.61-1.9)$ & 0.772 \\
\hline Tumor size $\geq 2$ cm & $47 / 166(28.3 \%)$ & $16 / 61(26.2 \%)$ & $0.90(0.46-1.7)$ & 0.756 \\
\hline Cervical stromal invasion & $24 / 176(13.6 \%)$ & $9 / 63(14.3 \%)$ & $1.1(0.46-2.4)$ & 0.898 \\
\hline Positive peritoneal cytology & $7 / 174(4.0 \%)$ & $11 / 63(17.5 \%)$ & $5.0(1.9-14)$ & 0.001 \\
\hline Age >65 years & $94 / 177(53.1 \%)$ & $44 / 64(68.8 \%)$ & $1.9(1.1-3.6)$ & 0.030 \\
\hline Body mass index $\geq 30 \mathrm{~kg} / \mathrm{m}^{2}$ & $65 / 177(36.7 \%)$ & $17 / 64(26.6 \%)$ & $0.62(0.33-1.2)$ & 0.141 \\
\hline
\end{tabular}

\footnotetext{
${ }^{\text {a }}$ Stage IV cancers excluded
} 
Table 3. Clinicopathological characteristics associated with L1CAM expression in preoperative endometrial samples, multivariate analysis

\begin{tabular}{|l|l|l|}
\hline Variable & OR $(95 \%$ CI $)$ & $\mathrm{P}$ \\
\hline Stage II-IV & $1.4(0.66-2.9)$ & 0.389 \\
\hline Non-endometrioid carcinoma & $4.4(1.4-14)$ & 0.010 \\
\hline Positive peritoneal cytology & $2.6(0.84-8.2)$ & 0.097 \\
\hline Age $>65$ years & $1.8(0.92-3.3)$ & 0.086 \\
\hline
\end{tabular}


Table 4. Risk factors associated with advanced (stage IIIC-IV) endometrial carcinoma, analysis by multivariate risk models. Patients with available data for all risk factors were included.

\begin{tabular}{|c|c|c|c|c|}
\hline & \multicolumn{2}{|c|}{ Models with L1CAM } & \multicolumn{2}{|c|}{ Models without L1CAM } \\
\hline & OR $(95 \% \mathrm{CI})$ & $\mathrm{P}$ & OR $(95 \% \mathrm{CI})$ & $\mathrm{P}$ \\
\hline Model HR-TD5cm-MI33\% & $1(n=225)$ & & $2(n=225)$ & \\
\hline Preoperative L1CAM & $4.1(1.5-11)$ & 0.007 & & \\
\hline Preoperative histology & $3.7(1.3-10)$ & 0.012 & $5.2(2.0-14)$ & 0.001 \\
\hline Tumor size $\geq 5 \mathrm{~cm}$ & $3.3(1.2-8.7)$ & 0.017 & $3.1(1.2-7.9)$ & 0.019 \\
\hline Myometrial invasion (MI) & & & & \\
\hline $\mathrm{MI} \leq 33 \%$ & 1 & & 1 & \\
\hline $33 \%<\mathrm{MI} \leq 66 \%$ & $3.3(0.72-15)$ & 0.125 & $3.0(0.67-14)$ & 0.151 \\
\hline $\mathrm{MI}>66 \%$ & $11(2.7-45)$ & 0.001 & $8.2(2.2-32)$ & 0.002 \\
\hline Model HR-TD5cm & $3(n=228)$ & & $4(n=228)$ & \\
\hline Preoperative L1CAM & $3.0(1.2-7.7)$ & 0.021 & & \\
\hline Preoperative histology & $4.8(1.8-13)$ & 0.002 & $6.6(2.7-17)$ & $<0.0001$ \\
\hline Tumor size $\geq 5 \mathrm{~cm}$ & $5.4(2.2-14)$ & $<0.0001$ & $5.0(2.1-12)$ & $<0.0001$ \\
\hline Model HR-TD2cm-MI50\% & $5(n=227)$ & & $6(n=227)$ & \\
\hline Preoperative L1CAM & $3.3(1.3-8.3)$ & 0.010 & & \\
\hline Preoperative histology & $4.3(1.7-11)$ & 0.003 & $5.8(2.3-15)$ & $<0.0001$ \\
\hline Tumor size $\geq 2 \mathrm{~cm}$ & $1.3(0.33-5.3)$ & 0.687 & $1.3(0.33-5.2)$ & 0.701 \\
\hline Myometrial invasion $\geq 50 \%$ & $4.9(1.7-14)$ & 0.003 & $4.3(1.5-12)$ & 0.007 \\
\hline Model HR-MI50\% & $7(n=241)$ & & $8(n=241)$ & \\
\hline Preoperative L1CAM & $3.0(1.2-7.4)$ & 0.015 & & \\
\hline Preoperative histology & $4.0(1.6-10)$ & 0.003 & $5.4(2.2-13)$ & $<0.0001$ \\
\hline Myometrial invasion $\geq 50 \%$ & $6.1(2.3-17)$ & $<0.0001$ & $5.3(2.0-14)$ & 0.001 \\
\hline
\end{tabular}

$\mathrm{HR}=$ high risk histology $(\mathrm{G} 3$ or non-endometrioid $) ; \mathrm{TD}=$ tumor diameter; $\mathrm{MI}=$ myometrial invasion 
Table 5. Areas under curve (AUC) for risk models predicting stage IIIC-IV endometrial carcinoma.

\begin{tabular}{|l|l|l|}
\hline Risk assessment model & AUC (95\% CI) & $\mathrm{P}$ (2-tailed) \\
\hline 1. HR-TD5cm-MI33\%-L1CAM & $0.879(0.828-0.930)$ & \\
\hline 2. HR-TD5cm-MI33\% & $0.870(0.813-0.928)$ & $\mathrm{P}=0.882$ vs. Model 1 \\
\hline 3. HR-TD5cm-L1CAM & $0.852(0.778-0.925)$ & \\
\hline 4. HR-TD5cm & $0.818(0.730-0.906)$ & $\mathrm{P}=0.613$ vs. Model 3 \\
\hline 5. HR-TD2cm-MI50\%-L1CAM & $0.841(0.777-0.905)$ & \\
\hline 6. HR-TD2cm-MI50\% & $0.805(0.717-0.894)$ & $\mathrm{P}=0.602$ vs. Model 5 \\
\hline 7. HR-MI50\%- L1CAM & $0.833(0.770-0.896)$ & \\
\hline 8. HR-MI50\% & & \\
\hline
\end{tabular}

$\mathrm{HR}=$ high risk histology $(\mathrm{G} 3$ or non-endometrioid $) ; \mathrm{TD}=$ tumor diameter; $\mathrm{MI}=$ myometrial invasion 